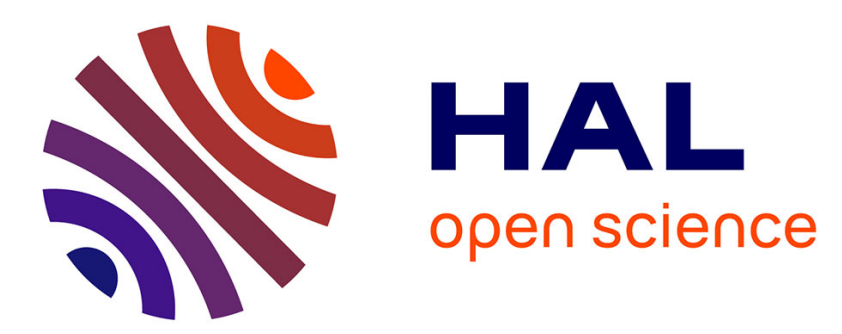

\title{
Computing the principal eigenvalue of the Laplace operator by a stochastic method
}

Antoine Lejay, Sylvain Maire

\section{To cite this version:}

Antoine Lejay, Sylvain Maire. Computing the principal eigenvalue of the Laplace operator by a stochastic method. Mathematics and Computers in Simulation, 2007, 73 (3), pp.351-363. 10.1016/j.matcom.2006.06.011 . inria-00092408

\section{HAL Id: inria-00092408 \\ https://hal.inria.fr/inria-00092408}

Submitted on 9 Sep 2006

HAL is a multi-disciplinary open access archive for the deposit and dissemination of scientific research documents, whether they are published or not. The documents may come from teaching and research institutions in France or abroad, or from public or private research centers.
L'archive ouverte pluridisciplinaire HAL, est destinée au dépôt et à la diffusion de documents scientifiques de niveau recherche, publiés ou non, émanant des établissements d'enseignement et de recherche français ou étrangers, des laboratoires publics ou privés. 


\title{
Computing the principal eigenvalue of the Laplace operator by a stochastic method
}

\author{
Antoine Lejay ${ }^{1, \dagger}$ — Projet OMEGA, INRIA \& Institut Élie Cartan, Nancy \\ Sylvain Maire ${ }^{2}$ - ISITV, Univ. de Toulon et du Var, Toulon
}

\begin{abstract}
We describe a Monte Carlo method for the numerical computation of the principal eigenvalue of the Laplace operator in a bounded domain with Dirichlet conditions. It is based on the estimation of the speed of absorption of the Brownian motion by the boundary of the domain. Various tools of statistical estimation and different simulation schemes are developed to optimize the method. Numerical examples are studied to check the accuracy and the robustness of our approach.
\end{abstract}

Keywords: First eigenvalue of the Dirichlet problem, Euler scheme for Brownian motion, random walk on spheres, random walk on rectangles

AMS Classification: 65C05, 60F 15

Published in Mathematics and Computers in Simulations.
In print

Archives, links \& reviews:

○ DOI: 10.1016/j.matcom.2006.06.011

${ }^{1}$ Current address: Projet OMEGA (INRIA/IECN)

IECN

Campus scientifique

BP 239

54506 Vandoeuvre-lès-Nancy CEDEX, France

E-mail: Antoine.Lejay@iecn.u-nancy.fr

${ }^{2}$ Current address: ISITV, Université de Toulon et du Var

Avenue G. Pompidou

BP 56

83262 La Valette du Var CEDEX, France

E-mail: maire@univ-tln.fr

${ }^{\dagger}$ Partially supported by GdR MOMAS (financed by the institutes ANDRA, BRGM, CEA, CNRS and EDF). 
A. Lejay and S. Maire / Computing the principal eigenvalue by Monte Carlo

\section{Introduction}

The aim of this paper is to introduce and study a Monte Carlo method for the numerical computation of the principal eigenvalue of the Laplace operator $\frac{1}{2} \triangle$ in a bounded domain $D \subset \Re^{d}$ with a sufficiently piecewise smooth boundary $\partial D$ and with Dirichlet boundary conditions. This leading eigenvalue determines the speed of convergence to the steady state for the solution of the heat equation. To compute it by a deterministic method, one has to discretize the Laplace operator using for example finite differences or finite elements and then evaluate the largest eigenvalue of the discretization matrix using for example the inverse power method. This computation becomes really expensive when the spatial dimension $d$ increases. Moreover the discretization should be refined enough so that the principal eigenvalue of the discretization matrix is close enough to the principal eigenvalue of the Laplace operator. Hence it is worth considering Monte Carlo methods [15] because they are usually efficient for this kind of difficult problems since they do not necessarily require to discretize the domain $D$ and they depend only linearly on the spatial dimension.

We have introduced in [17] a stochastic method to compute the principal eigenvalue of neutron transport operators based on the numerical computation of the type of the neutron transport operator. The idea was to combine the formal eigenfunction expansion of the solution of the relative Cauchy problem and its Monte Carlo evaluation via the Feynman-Kac formula. We intend to use the same methodology here in the case of the Laplace operator. The stochastic representation of the principal eigenvalue of the operator $\frac{1}{2} \triangle$ is usually achieved by combining the solution $u(t, x)$ of the Cauchy problem

$$
\left\{\begin{array}{l}
\frac{\partial u}{\partial t}=\frac{1}{2} \triangle u, \\
u(0, x) \equiv 1, x \in D \subset \mathbb{R}^{d}
\end{array}\right.
$$

obtained by the Feynman-Kac formula and the formal eigenfunction expansion

$$
u(t, x)=\sum_{j=1}^{\infty} c_{j} \exp \left(\lambda_{j} t\right) \Psi_{j}(x)
$$

of this solution in $\mathrm{L}^{2}(D)$ where $\lambda_{j}$ are the eigenvalues of $\frac{1}{2} \triangle$ arranged in decreasing order and $\Psi_{j}$ their relative eigenfunctions. Indeed as the solution of this equation is given by

$$
u(t, x)=\mathbb{P}\left(\tau_{D}^{x}>t\right),
$$

where $\tau_{D}^{x}$ is the exit time from $D$ of the Brownian motion starting at $x$, the principal eigenvalue $\lambda_{1}$ is directly linked to the speed of absorption of the 
A. Lejay and S. Maire / Computing the principal eigenvalue by Monte Carlo

Brownian motion by the boundary $\partial D$ and we have for all $x \in D$

$$
\lambda_{1}=\lim _{t \rightarrow+\infty} \frac{1}{t} \log \mathbb{P}\left(\tau_{D}^{x}>t\right) .
$$

This result is also true for a general elliptic operator $A$ with Dirichlet boundary conditions in a bounded domain $D$, where $\tau_{D}^{x}$ is the exit time from $D$ of the stochastic process $X_{t}^{x}$ generated by $A[7,8,13,19]$. We had to compute numerically the same quantity in [17] when studying homogeneous neutron transport operator. In this case, an exact simulation of transport processes involved in Feynman-Kac representations was possible. In the case of the Brownian motion, one has to use approximations based on various discretization schemes.

In Section 2, we describe quickly grid-free schemes, some of them common and some of them new, that can be used here. Then, we present in Section 3 different estimators for $\lambda_{1}$ based on an accurate study of the eigenfunctions expansion of the solution $u(t, x)$. Some of these estimators were developed in [17] but we also introduced new ones based on correlation coefficients. In order to compare the different simulations schemes and the different estimators, we study in detail in Section 4 a bidimensional problem. We finally study in Section 5 more difficult problems in dimension 3 and 5 combining all the tools developed previously in order to show that our method is also relevant in these cases.

\section{2 exit time simulation procedures}

The aim of this section is to describe and compare some simulation schemes for the exit time of the Brownian motion in a bounded domain $D$ with Dirichlet boundary conditions.

\section{$2.1 \quad$ Euler schemes}

The Euler scheme (see for example [14]) with discretization parameter $\Delta t$ writes

$$
X_{0}=x, X_{n+1}=X_{n}+\sqrt{\Delta t} Y_{n}
$$

where the $Y_{n}$ are independent standard Gaussian random variables. To compute $\mathbb{P}\left(\tau_{D}^{x}>t\right)$ one needs to compute simulations of $\tau_{D}^{x}$. With the crude version, the simulation stops once $X_{n+1} \in D^{\mathrm{c}}$ and $\tau_{D}^{x}$ is approximated either by $n \Delta t,\left(n+\frac{1}{2}\right) \Delta t$ or a slightly refined approximation based on the distances $d_{n}=d\left(X_{n}, \partial D\right)$ and $d_{n+1}=d\left(X_{n+1}, \partial D\right)$. In any case, these approximations are of weak order $\sqrt{\Delta t}$ and they overestimate this exit time. Indeed, the 
main simulation error does not come from the error at the last step but from the possibility for the Brownian motion to leave the domain between step $n$ and $n+1$ and be back into it at time $(n+1) \Delta t$. It is possible to take into account this possibility to obtain a scheme of weak order $\Delta t$ using the half-space approximation [9, 10]. An additional random test based on $d_{n}$ and $d_{n+1}$ is required. Taking a uniform random variable $U_{n}$, the motion stops if

$$
\exp \left(-\frac{2 d_{n} d_{n+1}}{\Delta t}\right)>U_{n} .
$$

It is still possible to introduce another refinement at the last step [3] which improves the accuracy but does not change the order of the scheme. In [11], another method is proposed that leads to the same order of convergence as in the half-space method. We only consider in the examples the naive version and the one using the half-space approximation, which we denote from now by the Improved Euler scheme.

\subsection{Walk on spheres (WOS)}

The simulation method based on the Euler schemes covers a wide range of elliptic and parabolic partial differential equations and can take into account the dependence of the drift and of the diffusion coefficients on the spatial variables. One only has to simulate a stochastic differential equation by means of this scheme instead of the Brownian motion. However, in the case of the heat equation some faster schemes are available. The walk on sphere schemes (WOS) 20] relies on the isotropy of the Brownian motion. This walk goes from $x$ to the boundary $\partial D$ from a sphere to another until the motion reaches an $\varepsilon$-absorption layer. The spheres are built so that the jumps are as large as possible. The radius of the next sphere from a starting point $x_{n}$ is $d\left(x_{n}, \partial D\right)$ and the next point is chosen uniformly on this sphere. The average number of steps to exit from the domain is proportional to $|\log (\varepsilon)|$ [20]. As we want here to simulate $\tau_{D}^{x}$, we need in addition to simulate the law $Y(r)$ of the exit time from a sphere of radius $r$ starting at its center. Some scaling arguments on the partial differential equation or on the Brownian motion show that $r^{2} Y(1)$ and $Y(r)$ have the same law. Some analytical expressions for the distribution function $H(t)=\mathbb{P}(Y(1)<t)$ are available. In dimension 2, we have for example [1]

$$
H(t)=\sum_{k=1}^{\infty} \frac{2 J_{0}(0)}{j_{k} J_{1}\left(j_{k}\right)} \exp \left(\frac{-j_{k}^{2} t}{2}\right)
$$

where $J_{0}$ and $J_{1}$ are Bessel functions and the $j_{k}$ are the positive zeros of $J_{0}$. The simulation of $Y(1)$ by the standard method requires the inversion of a 


\section{A. Lejay and S. Maire / Computing the principal eigenvalue by Monte Carlo}

series which can be difficult and costly. As we only need here to simulate $Y(1)$ during all the walk, we tabulate the distribution function once and for all and use it for simulations. Note that we can either use its analytical expression to do it or Monte Carlo simulations based on the corrected Euler scheme with a small parameter and a huge number of simulations. The second method is especially efficient in higher dimensions.

Some refined versions (for example, the walk on spheres with shifted centres [12]) allow faster absorption by the boundary, but also leads to simulate complex random variables.

\subsection{Walk on rectangles (WOR)}

In many practical situations (temperature evolution in a room, ...), the boundary is or can be approximated by a polygon. In this case, exact and fast simulations are possible.

The idea of the random walk on rectangles (WOR) is a generalization of the random walk on squares [4], that can be deduce from the algorithm given in [18] to simulate Stochastic Differential Equations (SDE). The idea is to simulate the exit time and position from a rectangle (or a parallelepiped in dimension greater than 2) by a Brownian motion. Unlike with a sphere, the random variables giving the exit time and position from a rectangle are not independent. Yet, as shown in [5], this could be achieved rather efficiently by proper conditioning and reducing the problem to simulate random variables related to the one-dimensional Brownian motion. The advantage of this method is that, unlike with the random walks of spheres and squares, the rectangles may be chosen prior to any simulation, since the Brownian motion can start from any point in it. In addition, it can be used even if a constant drift term is present, or to deal with Neumann boundary condition. Although it takes more time to simulate the exit time and position from a rectangle, the number of simulations is reduced. Of course, one has to use, when possible, rectangles for which at least one side corresponds to a boundary of $D$.

Let $\left(B^{1}, \ldots, B^{d}\right)$ be a $d$-dimensional Brownian motion with the starting point $\left(B_{0}^{1}, \ldots, B_{0}^{d}\right)=\left(x^{1}, \ldots, x^{d}\right)$. We denote by $\mathbb{P}_{x}$ the distribution of the one-dimensional Brownian motion starting at $x$. We are interested in simulating its first exit time and position from the parallelepiped $\left[-L_{1}, L_{1}\right] \times \cdots \times$ $\left[-L_{d}, L_{d}\right]$. For that, we set $\tau^{i}=\inf \left\{t>0 ; B_{t}^{i} \notin\left[-L_{i}, L_{i}\right]\right\}$ for $i=1, \ldots, d$ and we perform roughly the following operations:

(1) We draw a realization $\left(\theta^{1}, y^{1}\right)$ for $\left(\tau^{1}, B_{\tau^{1}}^{1}\right)$ under $\mathbb{P}_{x^{1}}$. We set $\bar{\tau}=\theta_{1}$, $S_{1}=1$ and $J=1$.

(2) For $i$ from 2 to $d$, do 
A. Lejay and S. Maire / Computing the principal eigenvalue by Monte Carlo

(2.1) We use a Bernoulli random variable to decide whether $\left\{\tau^{i}<\bar{\tau}\right\}$ or $\left\{\tau^{i} \geq \bar{\tau}\right\}$ under $\mathbb{P}_{x^{i}}$.

(2.2) If we have decided that $\left\{\tau^{i}<\bar{\tau}\right\}$, then we draw a realization $\left(\theta^{i}, y^{i}\right)$ of $\left(\tau^{i}, B_{\tau^{i}}^{i}\right)$ under $\mathbb{P}_{x^{i}}$ given $\left\{\tau^{i}<\bar{\tau}\right\}$. We set $J=i, S_{i}=1$ and $\bar{\tau}=\tau^{i}$.

(2.3) Otherwise, we set $S_{i}=0, \varphi_{i}=\bar{\tau}$.

(3) We set $z^{J}=y^{J}$.

(4) For $i$ from 1 to $d$, do

(4.1) If $S_{i}=1$ and $i \neq J$ then we simulate a realization $z^{i}$ of $B_{\bar{\tau}}^{i}$ under $\mathbb{P}_{x^{i}}$ given $\left\{\tau^{i}=\theta^{i}, B_{\tau^{i}}^{i}=y^{i}\right\}$.

(4.2) Otherwise, if $S_{i}=0$, then we simulate a realization $z^{i}$ of $B_{\bar{\tau}}^{i}$ under $\mathbb{P}_{x^{i}}$ given $\left\{\tau^{i} \geq \varphi_{i}\right\}$

(5) The algorithm stops and returns the time $\bar{\tau}$ and the position $\left(z^{1}, \ldots, z^{d}\right)$ on the boundary of the parallelepiped.

The involved distributions and the exact algorithm are detailed in [5].

\subsection{Walk on rectangles with importance sampling (WOR- IS)}

This method, developed in [6], is a variation on the previous method. Instead of simulating the exact couple exit time $\tau$ and exit position $B_{\tau}$ of a rectangle $R$ for a Brownian motion $B$, we draw this exit time and position $(\theta, Z)$ using an arbitrary distribution (of course, chosen to be simple), and we compute a weight $w$ such that $\mathbb{E}\left(f\left(\tau, B_{\tau}\right)\right)=\mathbb{E}(w f(\theta, Z))$ for any bounded, measurable function $f$ defined on $\mathbb{R}_{+} \times \partial R$. Of course, for any function $g$ defined on $\mathbb{R}_{+} \times \partial D$, the functional $\mathbb{E}\left(g\left(\tau_{D}^{x}, B_{\tau_{D}^{x}}\right)\right)$ is then evaluated by $\mathbb{E}\left(w_{1} \cdots w_{n^{*}} g\left(\theta_{n^{*}}, Z_{n^{*}}\right)\right)$, where the $\left(\theta_{i}, Z_{i}\right)$ 's are the successive exit times and positions for a sequence of rectangles, the $w_{i}$ 's are their associated weights, and $n^{*}$ is the first integer for which $Z_{n^{*}}$ belongs to $\partial D$, or is close enough to $\partial D$.

The advantage of this method over the WOR/WOS is that we are free to choose the distribution of $(\theta, Z)$, and then we can easily "constraint" the diffusion to go in some direction or condition it not to reach a part of the boundary of the domain. Thus, this method can be used to simulate Brownian motion or SDE in domains with complex geometries and to reduce the variance of the estimators that are computed. Moreover, this method is much 
more faster than the WOR if we choose for $(\theta, Z)$ random variables that are easy to simulate. In addition, the weights are rather easily computed since they rely on the density of the first exit time and the position of a killed Brownian motion in the one-dimensional case.

However, this method suffers from a severe drawback in our case. The empirical distribution function $F_{N}(t)$ of $F(t)=\mathbb{P}\left(\tau_{D}^{x}<t\right)$ is constructed as follows. If $\left(\theta^{(i)}, w^{(i)}\right)_{i=1, \ldots, N}$ are the simulated exit time from $D$ with their associated weights $w^{(i)}$, then

$$
F_{N}(t)=\frac{1}{N} \sum_{i=1}^{N} w^{(i)} \chi_{[0, t)}\left(\theta^{(i)}\right) .
$$

If $\widehat{F}^{N}(t)$ is the empirical distribution function constructed with $N$ independent random variables with the same law as $\tau_{D}^{x}$, then $\operatorname{Var}\left(\widehat{F}_{N}(t)\right)=$ $N^{-1} F(t)(1-F(t))$. In our case,

$$
\operatorname{Var}\left(F_{N}(t)\right)=\frac{1}{N} \operatorname{Var}\left(w^{(1)} \chi_{[0, t)}\left(\theta^{(1)}\right)\right) .
$$

The problem is then to find a "strategy" for which $\operatorname{Var}\left(F_{N}(t)\right)$ is smaller than $\operatorname{Var}\left(\widehat{F}_{N}(t)\right)$, at least when $t$ is large enough. Unfortunately, we have not been able to find a good way to do so. For the sake of simplicity, we have adopted the following strategy. For each rectangle, each of its side is reached with a probability $1 / 4$, the exit position is drawn using a uniform random variable on the side and the exit time is simulated using an exponential random variable of parameter 0.35 times the square of the length of the side that is not reached.

\section{Estimators of the principal eigenvalue}

\subsection{Direct approximation}

Using the formal eigenfunction expansion of $u(x, t)$, we can write that almost everywhere

$$
\frac{1}{t} \log \left(\mathbb{P}\left(\tau_{D}^{x}>t\right)\right)=\frac{1}{t} \log \left(C_{1} e^{\lambda_{1} t}+C_{2} e^{\lambda_{2} t}+\cdots+C_{k} e^{\lambda_{k} t}+\mathrm{o}\left(e^{\lambda_{k} t}\right)\right),
$$

where $C_{1}=c_{1} \Psi_{1}(x)$. The Krein-Rutman theorem [19] ensures that $\lambda_{1}$ is simple and $C_{1}>0$. Then

$$
\frac{1}{t} \log \mathbb{P}\left(\tau_{D}^{x}>t\right) \simeq \lambda_{1}+\frac{\log \left(C_{1}\right)}{t}+\frac{C_{2}}{C_{1}} \frac{e^{\left(\lambda_{2}-\lambda_{1}\right) t}}{t}
$$


A. Lejay and S. Maire / Computing the principal eigenvalue by Monte Carlo

keeping only the two dominant terms. The previous approximation shows that

$$
\lim _{t \rightarrow \infty} \frac{1}{t} \log \mathbb{P}\left(\tau_{D}^{x}>t\right)=\lambda_{1}
$$

almost everywhere and it has been proved in [13] that it holds everywhere. Hence the Monte Carlo computation of $t^{-1} \log \mathbb{P}\left(\tau_{D}^{x}>t\right)$ for large times offers a first possibility to give a numerical approximation of $\lambda_{1}$. Moreover we can choose the starting point wherever we want. The most natural choice is to take this starting point in the center of the domain in order to make the trajectories last as long as possible. Even if we do so, we will see in some numerical examples that this direct method gives in fact a poor approximation because the variance increases quickly with time. Indeed this involves the computation of the probability of rare events which become rarer and rarer as time increases. We now give some better estimators to overcome this difficulty.

\subsection{Interpolation method}

The previous expansion is constituted of a main term $\lambda_{1}+t^{-1} \log \left(C_{1}\right)$ and of a term $\frac{C_{2}}{C_{1}} \frac{e^{\left(\lambda_{2}-\lambda_{1}\right) t}}{t}$ which decays at an exponential rate the more quickly the first two eigenvalues are distant from each other. The difference $\lambda_{2}-\lambda_{1}$ is intrinsic to the domain. Nevertheless the choice of a starting point near the center of the domain makes the ratio $C_{2} / C_{1}$ smaller. This can be seen for instance on the eigenfunctions expansion on square domains. Moreover, it is well known that the errors due to the discretization scheme are smaller for points away from the boundary. If we assume that $t$ is large enough so that this term is negligible with respect to the others, we can compute easily $\lambda_{1}$ using the Monte Carlo approximations $\bar{p}_{1}$ and $\bar{p}_{2}$ of respectively $p_{1}=\mathbb{P}\left(\tau_{D}^{x, S}>t_{1}\right)$ and $p_{2}=\mathbb{P}\left(\tau_{D}^{x, S}>t_{2}\right)$ with a discretization scheme $S$. We obtain the estimators

$$
\lambda^{\mathrm{In}}\left(t_{1}, t_{2}\right)=\frac{\log \left(\bar{p}_{2}\right)-\log \left(\bar{p}_{1}\right)}{t_{2}-t_{1}}
$$

of $\lambda_{1}$ which are also used in neutron transport criticality computations [2]. Note that $\bar{p}_{1}$ and $\bar{p}_{2}$ are obviously correlated and may also be biased estimators of $\mathbb{P}\left(\tau_{D}^{x}>t_{1}\right)$ and $\mathbb{P}\left(\tau_{D}^{x}>t_{2}\right)$ because of the discretization errors. Letting $s_{1}=\sqrt{\bar{p}_{1}\left(1-\bar{p}_{1}\right)}$, we obtain the 95 percent confidence interval

$$
\bar{p}_{1}-1.96 \frac{s_{1}}{\sqrt{N}} \leq p_{1} \leq \bar{p}_{1}+1.96 \frac{s_{1}}{\sqrt{N}}
$$


that is assuming that $\frac{s_{1}}{\bar{p}_{1} \sqrt{N}}$ is small enough,

$$
\log \left(\bar{p}_{1}\right)-1.96 \frac{s_{1}}{\bar{p}_{1} \sqrt{N}} \leq \log \left(p_{1}\right) \leq \log \left(\bar{p}_{1}\right)+1.96 \frac{s_{1}}{\bar{p}_{1} \sqrt{N}}
$$

We finally have the 90 percent confidence interval for $\lambda^{\operatorname{In}}\left(t_{1}, t_{2}\right)$ writing

$$
\begin{aligned}
& \frac{\log \left(\frac{\bar{p}_{2}}{\bar{p}_{1}}\right)}{t_{2}-t_{1}}-\frac{1.96 N^{-\frac{1}{2}}}{t_{2}-t_{1}}\left(\frac{s_{1}}{\bar{p}_{1}}+\frac{s_{2}}{\bar{p}_{2}}\right) \\
& \quad \leq \lambda^{\operatorname{In}}\left(t_{1}, t_{2}\right) \leq \frac{\log \left(\frac{\bar{p}_{2}}{\bar{p}_{1}}\right)}{t_{2}-t_{1}}+\frac{1.96 N^{-\frac{1}{2}}}{t_{2}-t_{1}}\left(\frac{s_{1}}{\bar{p}_{1}}+\frac{s_{2}}{\bar{p}_{2}}\right) .
\end{aligned}
$$

\subsection{Least squares approximations}

In order to make a global use of the information given by the computation of the solution at the different times, we can give a least squares approximation of $\lambda_{1}$ and $\log \left(K_{0}\right)$ by fitting these parameters to the approximation model still assuming that the term $\frac{C_{2}}{C_{1}} \frac{e^{\left(\lambda_{2}-\lambda_{1}\right) t}}{t}$ is negligible. We denote by $F_{N}(t)$ be the empirical distribution of $\tau_{D}^{x}$, which is known at the set of times $t_{i}$. We have to find $\lambda_{1}$ and $\beta=\log \left(C_{1}\right)$ minimizing

$$
\sum_{i=p}^{q}\left(\lambda_{1}+\frac{\beta}{t_{i}}-\frac{1}{t_{i}} \log \left(1-F_{N}\left(t_{i}\right)\right)\right)^{2},
$$

which is a linear least squares problem with respect to the parameters $\lambda_{1}$ and $\beta$. This method was tested successfully in [16, 17] but we adopt a slightly different approach which appears to be more accurate and rigorous.

We now let $F(t)=\mathbb{P}\left(\tau_{D}^{x}<t\right)$ be the distribution function of the exit time for a fixed point $x$. Instead of computing $\lambda_{1}$ as the intersection value in the linear regression, we compute it as the slope of the regression line, since

$$
\log (1-F(t))=\lambda_{1} t+\log \left(c_{1} \Psi_{1}(x)\right)+\varepsilon(t)
$$

with

$$
\varepsilon=\log \left(1+R(t, x) / c_{1} \Psi_{1}(x)\right) \text { and } R(t, x)=\sum_{k \geq 2} e^{-\left(\lambda_{k}-\lambda_{1}\right) t} \Psi_{k}(x) c_{k} .
$$

Of course, in addition to $\varepsilon$, a second error comes from the replacement of $F(t)$ by an empirical density $F_{N}(t)$, where $N$ is the number of particles in the simulation. Thus, we are indeed using a linear regression for the equation

$$
\log \left(1-F_{N}(t)\right)=-\lambda_{1} t+\log \left(\alpha_{1} \Psi_{1}(x)\right)+\varepsilon(t)+\eta_{N}(t)
$$


with

$$
\eta_{N}(t)=\log \left(1-\frac{F(t)-F_{N}(t)}{1-F(t)}\right) \approx \frac{F_{N}(t)-F(t)}{1-F(t)} .
$$

The speed of convergence of $F_{N}(t)$ to $F(t)$ given by the Kolmogorov-Smirnov test [21] is of order $1 / \sqrt{N}$. We will see that our estimate of $\lambda_{1}$ is more likely to be limited by $\eta_{N}$, and thus by the number $N$ of samples than by $\varepsilon$.

We can be even more precise. The empirical distribution function $F_{N}(t)$ converges uniformly to $F(t)$. Moreover, $\sqrt{N}\left(F_{N}(t)-F(t)\right)$ converges in distribution to a Gaussian process $\left(x_{t}\right)_{t \geq 0}$ with $\operatorname{Var}\left(x_{t}\right)=F(t)(1-F(t))$ and $\operatorname{Cov}\left(x_{t}, x_{s}\right)=F(s)(1-F(s))$ when $t \geq s$. This implies that

$$
\operatorname{Var}\left(\eta_{N}(t)\right) \approx \frac{1}{N} \frac{F(t)}{1-F(t)}
$$

which was already used in the interpolation method. Thus, a good estimate of $\lambda_{1}$ relies on using a window in which both $\varepsilon(t)$ (which is unknown) and $\operatorname{Var}\left(\eta_{N}(t)\right)$ are small enough.

Remark 1 . We could also use weighted least squares methods with weights given by $\left(1-F_{N}(t)\right) / F_{N}(t)$ in order to compensate the variance of the $\eta_{N}(t)$. In practice, we have noticed no real improvement.

\section{A 2D test case}

\subsection{Description}

We deal with the 2 dimensional domain seen in Figure 1

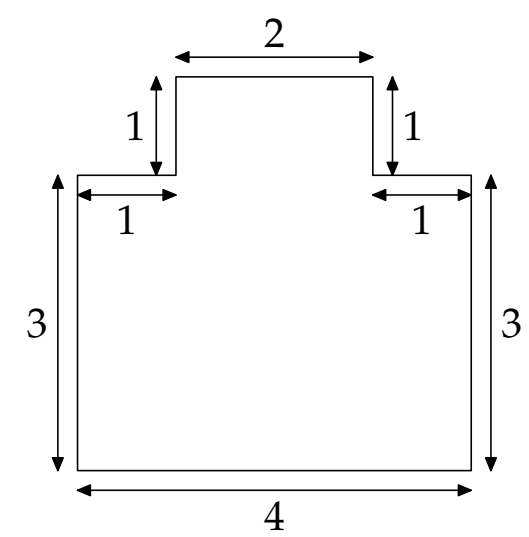

Figure 1: The domain $D$ for the 2D test case 
First, we estimate the first eigenvalue $\lambda_{2 D}$ of $\frac{1}{2} \triangle$ using the pdetool package from MATLAB. In Table 1, we give the estimates of $\lambda_{2 D}$ as a function of the number of times we refine the mesh. Thus, its seems that the real value of $\lambda_{2 D}$ is close to -0.740 . The order of the second eigenvalue is -1.75 .

\begin{tabular}{|c|c|c|c|c|c|c|}
\hline \# of nodes & 180 & 670 & 2590 & 10200 & 40400 & 160940 \\
\hline$-\lambda_{2 D}$ & 0.7578 & 0.74532 & 0.74415 & 0.74021 & 0.73975 & 0.73958 \\
\hline
\end{tabular}

Table 1: Estimate by MATLAB/pdetool in function of the refinement of the mesh

\subsection{The empirical distribution function of the first exit time by Monte Carlo methods}

We have considered the simulation of the function $F(t)$ using the different schemes described in Section 2 with 1.000.000 particles, excepted for the WOR-IS where 100.000.000 particles were used (see Section 2.4 for an explanation). For the Euler scheme, we use a time step $\Delta t$ of $10^{-3}$. For the walk on spheres, the absorption boundary layer is $\varepsilon=10^{-3}$. For the WOR and WOR-IS, we use only the two rectangles $[0,4] \times[0,3]$ and $[1,3] \times[0,4]$ (the lower left corner is at $(0,0)$ ). The computation times are presented in Section 4.5.

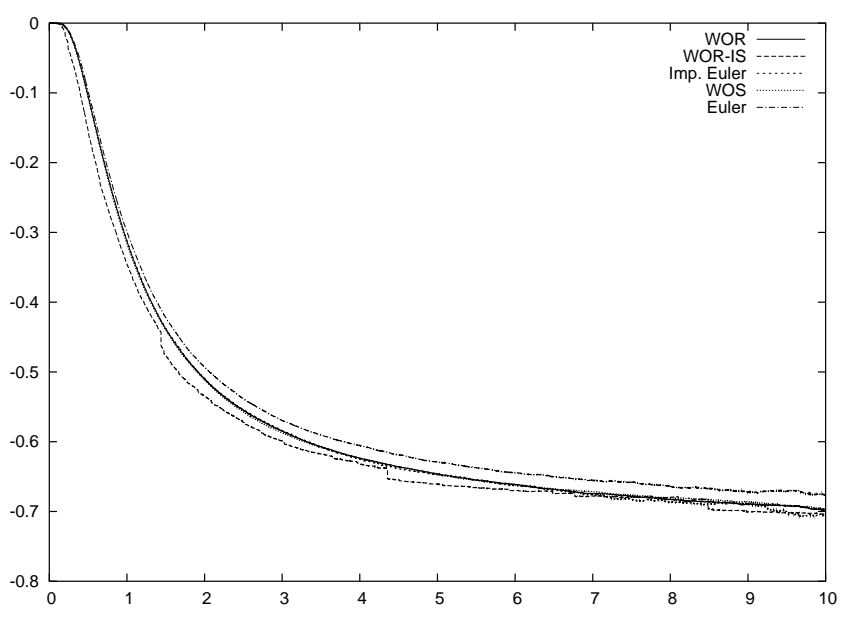

Figure 2: $t^{-1} \log \left(1-F_{N}(t)\right)$ for the different schemes

In Figure 2, we have drawn $G_{N}(t)=t^{-1} \log \left(1-F_{N}(t)\right)$ where the starting point is $(2,2)$. All the schemes give a function $G_{N}$ decreasing to a value 
which is around -0.7 at $t=10$, except for the naive Euler scheme for which it is around -0.67 . For the WOR-IS, we have tested different strategies to force the process to stay longer times in the domain, but none were very successful. In addition, we see that the curves are perturbed by numerical artifacts.

Yet for large times, that is $t>7.0$, the behavior becomes quite erratic, since it corresponds to the simulation of rare events. We cannot expect to obtain an accurate estimate of $\lambda_{2 D}$ this way.

\subsection{Estimation by the interpolation method}

We then use the interpolation method of Section 3.2, with $t_{2}-t_{1}=4$ and we show in Figure 3 the values of $\lambda_{1}^{S}\left(t_{1}, t_{2}\right)$ for $t_{1} \in[2,5]$.

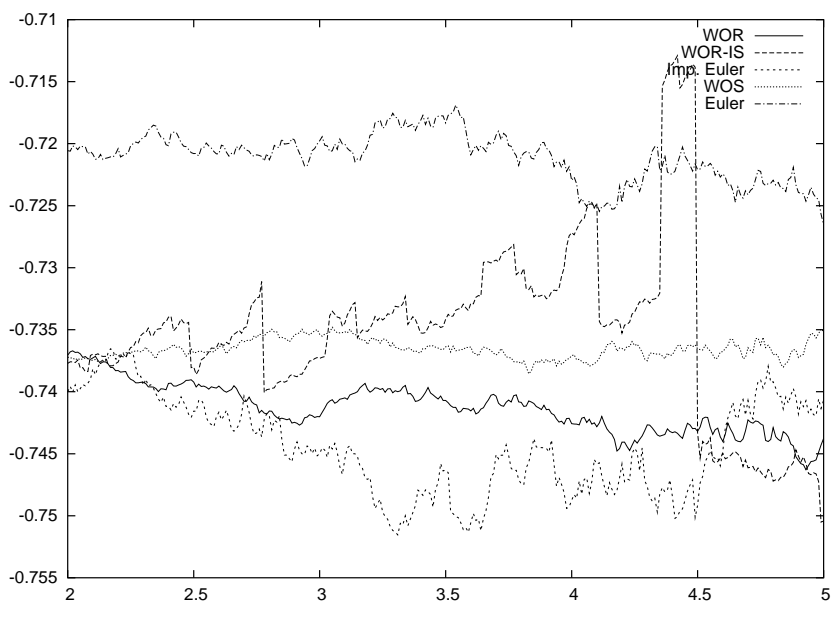

Figure 3: The estimator $\lambda^{\operatorname{In}}\left(t_{1}, t_{1}+4\right)$ for the different schemes

We obtain more accurate estimators than with the previous method. We still observe a deterioration when $t_{1}$ is too large for all the schemes. However, the naive Euler scheme gives an overestimated value of $\lambda_{2 D}$, which can be easily explained by its nature. We no longer consider this scheme in the following.

If we look for the value of $\lambda^{\mathrm{In}}\left(t_{1}, t_{1}+4\right)$ with $t_{1}$ in some particular intervals, e.g. $t_{1} \in[2,3]$, we can see that all the estimators are very close to -0.74 and this for all the remaining schemes. We now turn to more quantitative results. 
A. Lejay and S. Maire / Computing the principal eigenvalue by Monte Carlo

\subsection{Optimization of the estimators}

To estimate $\lambda_{2 D}$, we are free to choose the range of time $t$ for $F(t)$. The idea is then to consider an estimator $\lambda_{2 D}^{(i)}$ of $\lambda_{2 D}$ using the data on an interval $\left[a_{i}, b_{i}\right]$ for a large choice of $a_{i}$ and $b_{i}$. We perform a statistical analysis of the set of the $\lambda_{2 D}^{(i)}$ for which a quality criterion is satisfied.

We first use the confidence interval techniques of Section 3.2 after having remarked on Figure 3 that all the estimators $\lambda^{\operatorname{In}}\left(t_{1}, t_{1}+4\right)$ are contained in a narrow band for $t_{1} \in[2,5]$. We now compute all the possible estimators $\lambda^{\operatorname{In}}\left(t_{1}, t_{2}\right)$ and their relative confidence intervals, where $t_{1}, t_{2}$ are chosen on a grid $\eta \mathbb{Z}$ with $\eta=1 / 10, t_{1} \geq 2, t_{2} \leq 8$ and $t_{2}-t_{1} \geq 2$. We only keep the fraction of these estimators for which the length of the confidence interval is small. Indeed, we have to find a balance in the length $\ell_{90 \%}\left(t_{1}, t_{2}\right)$ of the confidence interval at $90 \%$ given by (1) between the term $1 /\left(t_{2}-t_{1}\right)$ which shall be small and the term $s_{1} / \bar{p}_{1}+s_{2} / \bar{p}_{2}$ that increases when $t_{1}$ or $t_{2}$ increases. We plot $\lambda^{\operatorname{In}}\left(t_{1}, t_{2}\right)$ against $\ell_{90 \%}\left(t_{1}, t_{2}\right)$ and we remark that the quality of the approximation decreases when $\ell_{90 \%}\left(t_{1}, t_{2}\right)$ increases.

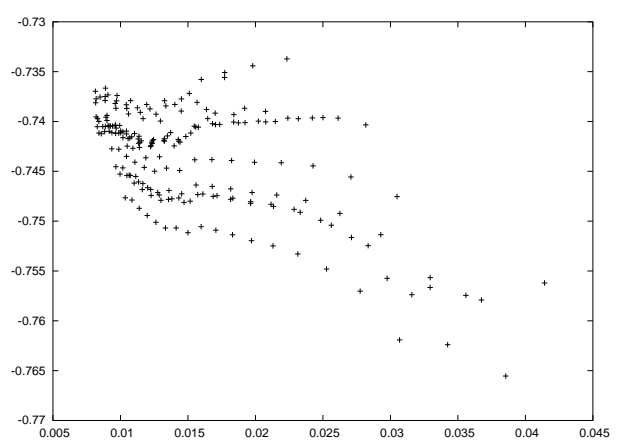

Figure 4: $\lambda^{\operatorname{In}}\left(t_{1}, t_{2}\right)$ in function of $\ell_{90 \%}\left(t_{1}, t_{2}\right)$ for the improved Euler scheme

We also note that the smallest values of $\ell_{90 \%}\left(t_{1}, t_{2}\right)$ are obtained when $t_{1}$ is close to 2 and $t_{2}$ close to 5 .

We now present our results on Table 2 using classical statistical estimators for the $\lambda^{\operatorname{In}}\left(t_{1}, t_{2}\right)$ 's satisfying our criterion. 
A. Lejay and S. Maire / Computing the principal eigenvalue by Monte Carlo

\begin{tabular}{ccccc}
\hline Method & Improved Euler & WOS & WOR & WOS-IS \\
\hline Min. & -0.7423 & -0.7385 & -0.7398 & -0.7537 \\
1st Qu. & -0.7403 & -0.7378 & -0.7376 & -0.7456 \\
Median & -0.7392 & -0.7373 & -0.7373 & -0.7393 \\
Mean & -0.7391 & -0.7372 & -0.7374 & -0.7404 \\
3rd Qu. & -0.7377 & -0.7368 & -0.7369 & -0.7364 \\
Max & -0.7365 & -0.7351 & -0.7361 & -0.7268 \\
\hline
\end{tabular}

Table 2: Study of the estimators $\lambda^{\operatorname{In}}\left(t_{1}, t_{2}\right)$ by keeping the $10 \%$ having the smallest value of $\ell_{90 \%}\left(t_{1}, t_{2}\right)$

If we look at the median or the mean of the estimators $\lambda^{\operatorname{In}}\left(t_{1}, t_{2}\right)$, we can reach an accuracy of at least $3 \cdot 10^{-3}$. All the schemes give an analogous accuracy.

We now turn to the least squares method, by replacing our criterion on the length of the confidence interval by a criterion based on the correlation coefficient $R$. This coefficient measures the validity of the linear approximation. We denote by $\lambda^{\mathrm{LS}}\left(t_{1}, t_{2}\right)$ the estimator of $\lambda^{\mathrm{LS}}\left(t_{1}, t_{2}\right)$ giving the slope of the linear regression of $G_{N}(t)$ with $t \in\left[t_{1}, t_{2}\right]$. We select the estimators $\lambda^{\mathrm{LS}}\left(t_{1}, t_{2}\right)$ for which $R^{2}$ is greater than 0.99997 . We also plot $\lambda^{\mathrm{LS}}\left(t_{1}, t_{2}\right)$ against the coefficient $R^{2}$. We now present our results on $\lambda^{\mathrm{LS}}\left(t_{1}, t_{2}\right)$ in Table 3 as we did previously for $\lambda^{\operatorname{In}}\left(t_{1}, t_{2}\right)$.

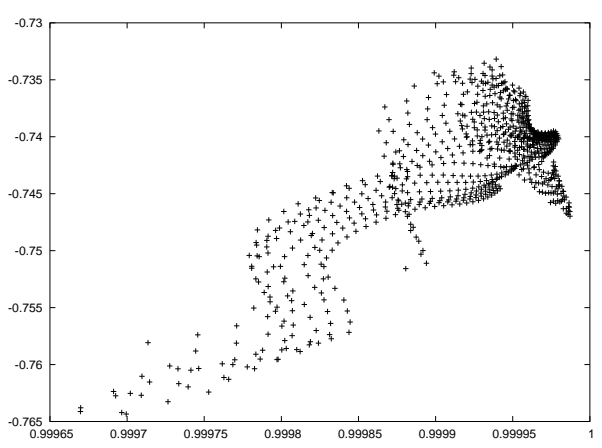

Figure 5: $\lambda^{\mathrm{LS}}\left(t_{1}, t_{2}\right)$ in function of $R^{2}$ for the improved Euler scheme 
A. Lejay and S. Maire / Computing the principal eigenvalue by Monte Carlo

\begin{tabular}{cccc}
\hline Method & Improved Euler & WOS & WOR \\
\hline Min. & -0.7470 & -0.7415 & -0.7443 \\
1st Qu. & -0.7444 & -0.7375 & -0.7411 \\
Median & -0.7413 & -0.7368 & -0.7396 \\
Mean & -0.7423 & -0.7367 & -0.7398 \\
3rd Qu. & -0.7401 & -0.7360 & -0.7380 \\
Max & -0.7395 & -0.7321 & -0.7370 \\
\hline
\end{tabular}

Table 3: Study of the estimators $\lambda^{\mathrm{LS}}\left(t_{1}, t_{2}\right)$ for $R^{2}>0.99997$

Once again, we obtained a good accuracy on the first eigenvalue $\lambda_{2 D}$ (at least $3 \cdot 10^{-3}$ ). However, for the WOR-IS, this criterion is less robust and we have obtained an accuracy $3 \cdot 10^{-2}$ and thus we do not include the results in Table 3 .

\subsection{Computation times}

Although the schemes give close estimated values of $\lambda_{2 D}$, their relative computation times differ largely and may be function of the choice of some parameters, as seen in Table 4. The simulations were done on a bi-processors DEC $700 \mathrm{MHz}$.

\begin{tabular}{ccccc}
\hline Scheme & Parameter name & Parameter & Time $(\mathrm{s})$ & Avg. \# steps \\
\hline \multirow{2}{*}{ Euler } & \multirow{2}{*}{ time step $\Delta t$} & $10^{-2}$ & 210 & 200 \\
& & $10^{-3}$ & 1400 & 2000 \\
\hline \multirow{2}{*}{ WOS } & \multirow{2}{*}{ boundary layer $\varepsilon$} & $10^{-3}$ & 80 & 13 \\
& & $10^{-4}$ & 85 & 16 \\
\hline WOR & - & - & 700 & 1.5 \\
\hline WOR-IS & - & - & 16 & 2.1 \\
\hline
\end{tabular}

Table 4: Computations times with $N=1.000 .000$ for all the methods

We compare first the average number of steps of each of the methods. The WOR and WOR-IS take around two steps to reach the boundary. The WOS takes around ten times more steps using a boundary layer $\varepsilon=10^{-4}$. This number of steps increases slowly like a $\mathrm{O}(|\log (\varepsilon)|)$ as $\varepsilon$ goes to zero. The Euler scheme with $\Delta t=10^{-2}$ takes ten times more steps than the WOS. This number of steps increases linearly with $\Delta t$ (if $\Delta t$ is divided by 10 the number of steps is ten times greater). If we now look at the CPU times, the WOR-IS is really the fastest method as its average number of steps is 2 and that it 
A. Lejay and S. Maire / Computing the principal eigenvalue by Monte Carlo

requires the simulation of fairly simple random variables. Unfortunately, this method increases somehow the variance of the simulations. A lot more samples are required to make it as accurate as the other methods. The simulation times of the WOR and of the Euler scheme are comparable. The first one requires only 1.5 steps but the simulation of complex random variables. The second one requires many steps but the simulation of very simple Gaussian random variables. A good arrangement between these methods appears to be the WOS. The average number of steps is small and the random variables to simulate are not too complex.

\subsection{Preliminary conclusions}

Our approach is efficient on this test case. However, among the schemes we have tested, the naive Euler scheme shall be discarded. Our statistical study gives an approximation of $\lambda_{2 D}$ with an accuracy of order $3 \cdot 10^{-3}$, which corresponds to what can be expected from the replacement of $F(t)$ by an empirical distribution function, due to the Kolmogorov-Smirnov theorem.

In practice, we recommend the two following methods:

(a) We look graphically at either $\lambda^{\operatorname{In}}\left(t_{1}, t_{1}+\tau\right)$ for a given $\tau$ and $t_{1}$ in the range of the obtained values and we then determine a range of times for which the oscillations around a constant value are small enough. We may use either the least squares method or the interpolation method.

(b) We use the least squares methods to estimate $\lambda^{\mathrm{LS}}\left(t_{1}, t_{2}\right)$ for a large number of values of $t_{1}<t_{2}$ with $t_{2}-t_{1} \geq \tau$ for an arbitrary $\tau$, and keep the values of $\lambda^{\mathrm{LS}}\left(t_{1}, t_{2}\right)$ for which the coefficient $R^{2}$ is large enough.

\section{More complex problems}

\subsection{A three dimensional problem}

We now consider the extension of our 2D problem where we add a third dimension $z \in[0,4]$. We could treat as well more complex three dimensional domains but we use this problem as its main eigenvalue can be simply computed. By a separation of variables argument, we have $\lambda_{2 D}-\frac{\pi^{2}}{32} \simeq-1.048$.

In Table 5, we use confidence intervals and least squares approximations for intervals $\left[t_{1}, t_{2}\right]$ contained in $[2,8]$ with $t_{2}-t_{1} \geq 2$ and a step of 0.1 for $t_{1}$ and $t_{2}$. 
A. Lejay and S. Maire / Computing the principal eigenvalue by Monte Carlo

\begin{tabular}{|c|cc|cc|}
\hline Method & \multicolumn{2}{|c|}{ WOR } & \multicolumn{2}{c|}{ WOS } \\
\hline \hline Parameter & \multicolumn{2}{|c|}{-} & \multicolumn{2}{c|}{$\varepsilon=10^{-4}$} \\
\hline Time (s) & \multicolumn{2}{|c|}{1200} & \multicolumn{2}{|c|}{110} \\
\# steps & \multicolumn{2}{|c|}{1.34} & \multicolumn{2}{|c|}{30} \\
\hline & $\lambda^{\mathrm{LS}}$ & $\lambda^{\mathrm{In}}$ & $\lambda^{\mathrm{In}}$ \\
\hline$R_{\min }^{2}$ or $\ell_{\max }$ & 0.99995 & 0.02 & 0.99995 & 0.02 \\
\# samples & 141 & 125 & 695 & 135 \\
\hline Min. & -1.056 & -1.048 & -1.049 & -1.049 \\
1st Qu. & -1.049 & -1.045 & -1.047 & -1.048 \\
Median & -1.046 & -1.044 & -1.047 & -1.047 \\
Mean & -1.047 & -1.044 & -1.046 & -1.047 \\
3rd Qu. & -1.045 & -1.043 & -1.046 & -1.046 \\
Max & -1.041 & -1.042 & -1.041 & -1.045 \\
\hline
\end{tabular}

Table 5: $\quad$ Study of the estimators $\lambda^{\operatorname{In}}\left(t_{1}, t_{2}\right)$ for $\ell_{90 \%}\left(t_{1}, t_{2}\right) \leq \ell_{\max }$ and $\lambda^{\mathrm{LS}}\left(t_{1}, t_{2}\right)$ for $R^{2} \geq R_{\min }^{2}$ with $N=1.000 .000$ particles.

We focus on this example on the WOR ans WOS methods. For the WOS, we need to simulate the uniform law on the unit sphere. This density writes

$$
f(\theta, \varphi)=\frac{1}{2} \sin (\theta) \mathbf{1}_{[0, \pi)}(\theta) \frac{1}{2 \pi} \mathbf{1}_{[0,2 \pi)}(\varphi) .
$$

The three Cartesian coordinates are

$$
x=\sin (\theta) \sin (\varphi), y=\sin (\theta) \cos (\varphi), z=\cos (\theta)
$$

where the density of $\theta$ is $\frac{1}{2} \sin (\theta) \mathbf{1}_{[0, \pi)}(\theta)$ and where $\varphi$ is uniform on $[0,2 \pi)$. The simulation of $\theta$ is achieved using the standard method by $2 \arcsin (\sqrt{U})$ where $U$ is uniform on $[0,1)$. The distribution function of the exit time from the unit ball is obtained via Monte Carlo simulations.

We note that once again we obtain an accuracy of about three digits on the principal eigenvalue using either the WOR or the WOS method. The two statistical methods lead to very similar approximations. If we look at the CPU times, we observe that they increase only linearly with the dimension of the problem.

\subsection{A five dimensional problem}

We now take some more examples in dimension 5 to emphasize that our method is not very sensitive to the dimensional effect. Similarly to the 3D case, we consider the Dirichlet problem on the domain

$$
D_{5 D}=\left\{\left(x_{1}, \ldots, x_{5}\right) \in \mathbb{R}^{5} ;\left(x_{1}, x_{2}\right) \in D,\left(x_{3}, x_{4}, x_{5}\right) \in[0,4]^{3}\right\} .
$$


A. Lejay and S. Maire / Computing the principal eigenvalue by Monte Carlo

Using again a separation of variables, the first eigenvalue $\lambda_{5 D}$ is $\lambda_{2 D}-$ $3 \pi^{2} / 32 \simeq-1.665$, if $\lambda_{2 D}$ is approximated by -0.740 .

In Table 6, we use confidence intervals and least squares approximations for intervals $\left[t_{1}, t_{2}\right]$ contained in $[2,6]$ with $t_{2}-t_{1} \geq 2$ and a step of 0.1 .

\begin{tabular}{|c|cc|cc|cc|}
\hline Method & \multicolumn{2}{|c|}{ WOR } & \multicolumn{2}{c|}{ Imp. Euler } & \multicolumn{2}{c|}{ Imp. Euler } \\
\hline \hline Parameter & \multicolumn{2}{|c|}{-} & \multicolumn{2}{c|}{$\Delta t=10^{-2}$} & \multicolumn{2}{c|}{$\Delta t=6 \cdot 10^{-3}$} \\
\hline Time (s) & \multicolumn{2}{|c|}{2000} & \multicolumn{2}{c|}{300} & \multicolumn{2}{c|}{440} \\
\# steps & \multicolumn{2}{|c|}{1.23} & \multicolumn{2}{c|}{123} & \multicolumn{2}{c|}{200} \\
\hline & $\lambda^{\mathrm{LS}}$ & $\lambda^{\mathrm{In}}$ & $\lambda^{\mathrm{In}}$ & $\lambda^{\mathrm{LS}}$ & $\lambda^{\mathrm{In}}$ \\
\hline$R_{\min }^{2}$ or $\ell_{\max }$ & 0.99995 & 0.06 & 0.9999 & 0.06 & 0.9999 & 0.06 \\
\# samples & 20 & 56 & 140 & 62 & 90 & 64 \\
\hline Min. & -1.666 & -1.682 & -1.692 & -1.677 & -1.667 & -1.673 \\
1st Qu. & -1.663 & -1.677 & -1.676 & -1.671 & -1.663 & -1.666 \\
Median & -1.662 & -1.672 & -1.671 & -1.668 & -1.658 & -1.657 \\
Mean & -1.661 & -1.672 & -1.671 & -1.667 & -1.658 & -1.658 \\
3rd Qu. & -1.659 & -1.666 & -1.667 & -1.665 & -1.653 & -1.650 \\
Max & -1.654 & -1.662 & -1.651 & -1.657 & -1.646 & -1.645 \\
\hline
\end{tabular}

Table 6: Study of the estimators $\lambda^{\mathrm{In}}\left(t_{1}, t_{2}\right)$ for $\ell_{90 \%}\left(t_{1}, t_{2}\right) \leq \ell_{\max }$ and $\lambda^{\mathrm{LS}}\left(t_{1}, t_{2}\right)$ for $R^{2} \geq R_{\min }^{2}$ with $N=1.000 .000$ particles.

As the WOS is not so easy to implement in this case, we now use the WOR and the improved Euler scheme in the simulations. We still obtain in less than 10 minutes of CPU a good accuracy of about $3 \cdot 10^{-3}$ on the eigenvalue for a problem which is very difficult to solve by means of deterministic methods. The CPU times of the WOR still increases linearly. It seems sufficient to use a discretization parameter of $\Delta t=10^{-2}$ for the Euler Scheme to reach this accuracy. We can certainly recommend this method for such difficult problems as it is very easy to implement.

\section{Conclusion}

We have presented and tested a Monte Carlo method to compute the first eigenvalue $\lambda_{1}$ of the Laplace operator with Dirichlet boundary condition. This method is based on the numerical computation of the speed of absorption of the Brownian motion by the boundary of the domain. It requires a good approximation of the law of the exit time of the Brownian motion by means of various schemes and also accurate estimators of $\lambda_{1}$ seen as a parameter of a model. 
A. Lejay and S. Maire / Computing the principal eigenvalue by Monte Carlo

We have to note that the estimators of $\lambda_{1}$ are very sensitive to the quality of the empirical distribution function of the exit time. In our least squares method, we have however developed a test based on the coefficient $R^{2}$, which appears to be robust in all our experiments. The accuracy is anyhow limited by the speed of convergence given by the Kolmogorov-Smirnov theorem.

The WOR-IS is not efficient in our case, while the naive Euler scheme overestimates $\lambda$ and is also to be rejected for this purpose. Yet some variants of the Euler scheme provide much more better estimates, while remaining easy to set up. The WOS is the fastest method there, but may be difficult to set up in high dimension. For polytopes, the WOR provides a good approximation of $\lambda_{1}$ but takes longer time in high dimension, and is more difficult to implement. Note that however partial tabulations of some random variables and a better use of the WOR-IS should provide very efficient methods.

On all our test cases, from dimension 2 to 5 , we have obtained in no more than $10 \mathrm{~min}$ an accuracy of about 3 digits on the eigenvalue $\lambda_{1}$ using 1.000.000 particles. Of course, in our test cases, the geometry of the domain was rather simple, but the different methods can be combined to deal with more complex geometries. We can also add that our method is even more valuable in dimension greater than 3 , where deterministic methods are limited by the difficulty of generating a mesh and the size of the matrix to invert. In all our methods, the computation time increases linearly with the dimension, and none requires the construction of a mesh. Besides, this method can be extended to deal with a general second-order elliptic operator. The computation of $\lambda_{1}$ could also be a good test to evaluate the efficiency of diffusion approximations.

\section{References}

[1] A. Borodin and P. Salminen, Handbook of Brownian motion: facts and formulae. Birkhäuser, 1996. 4

[2] D. Brockway, P. Soran and P. Whalen. Monte Carlo eigenvalue calculation, in Monte-Carlo methods and applications in neutronics, photonics and statistical physics. R. Alcouffe et al. (Eds), 378-384, Springer-Verlag, 1985. 8

[3] F. M. Buchmann. Simulations of stopped diffusions, J. Comput. Phys. 202, no. 2, 446-462, 2005. 4 
A. Lejay and S. Maire / Computing the principal eigenvalue by Monte Carlo

[4] F. Campillo and A. Lejay. A Monte Carlo method without grid for a fractured porous domain model, Monte Carlo Methods Appl., 8, no. 2, 129-148, 2002. 5

[5] M. Deaconu and A. Lejay. A random walk on rectangles algorithm. Methodol. Comput. Appl. Probab., 8, no. 1, 135-151, 2006. 5, 6

[6] M. Deaconu and A. Lejay. Simulation of a diffusion process using the importance sampling paradigm. In preparation, 2005. 6

[7] M. Freiduin. Functional Integration and Partial Differential Equations. Princeton University Press, 1985. 3

[8] A. Friedman. Stochastic Differential Equations and Applications, Volume 2, Academic Press, 1976. 3

[9] E. Gobet. Weak approximations of killed diffusions using Euler schemes. Stoch. Process. Appl., 87, no. 2, pp 167-197, 2000. 4

[10] E. Gobet. Euler schemes and half-space approximations for the simulation of diffusion in a domain, ESAIM Probability and Statistics, 5, 261-297, 2001. 4

[11] E. Gobet and S. Menozzi. Exact approximation rate of killed hypoelliptic diffusions using the discrete Euler scheme, Stochastic Process. Appl., 112, no. 2, 201-223, 2004. 4

[12] N. Golyandina. Convergence rate for spherical processes with shifted centres. Monte Carlo Methods Appl. 10, no. 3-4, 287-296, 2004. 5

[13] Y. KIFER. Random perturbations of dynamical systems. Progress in Probability and Statistics, 16. Birkhäuser, 1988. 3, 8

[14] P. Kloeden and E. Platen. Numerical solution of stochastic differential equations. Applications of Mathematics, Springer-Verlag, Berlin, 1992. 3

[15] B. Lapeyre, E. Pardoux and R. Sentis. Méthodes de Monte-Carlo pour les équations de transport et de diffusion. Springer-Verlag, 1998. 2

[16] S. MAIRE. Réduction de variance pour l'intégration numérique et pour le calcul critique en transport neutronique, Phd thesis, Université de Toulon et du Var, 2001. 9 
A. Lejay and S. Maire / Computing the principal eigenvalue by Monte Carlo

[17] S. Maire and D. TAlay. On a Monte Carlo method for neutron transport criticality computations. IMA J. Numer. Anal., to appear, DOI:10.1093/imanum/dr1008, 2006. 2, 3, 9]

[18] G. Milstein and M. Tretyakov. Simulation of a space-time bounded diffusion. Ann. Appl. Probab., 9, no. 3, 732-779, 1999. 5

[19] R. G. Pinsky. Positive harmonic functions and diffusion. Cambridge Studies in Advanced Mathematics, 45. Cambridge University Press, 1995. 3, 7

[20] K. SABelfeld. Monte Carlo methods in boundary value problems. Springer, 1991. 4

[21] G. ShORACK and J. WelLner. Empirical processes with applications to statistics. Wiley Series in Probability and Mathematical Statistics: Probability and Mathematical Statistics. John Wiley \& Sons, 1986. 10 